\title{
Quasiconvex stochastic processes and a separation theorem
}

\author{
Dawid Kotrys and KaZimierz Nikodem \\ Dedicated to Professor János Aczél on his $90^{\text {th }}$ birthday
}

\begin{abstract}
Quasiconvex stochastic processes are introduced. A characterization of pairs of stochastic processes that can be separated by a quasiconvex stochastic process and a stability theorem for quasiconvex processes are given.
\end{abstract}

Mathematics Subject Classification (2010). Primary: 26A51 · Secondary: 60G99.

Keywords. Quasiconvex stochastic process - Separation by quasiconvex stochastic processes $\cdot$ Stability of quasiconvex stochastic processes.

\section{Introduction}

In [1], Baron et al. proved that two real functions $f$ and $g$ defined on a real interval $I$ can be separated by a convex function if and only if they fulfil the following inequality

$$
f(\lambda x+(1-\lambda) y) \leqslant \lambda g(x)+(1-\lambda) g(y),
$$

for all $x, y \in I$ and $\lambda \in[0,1]$.

In 1994 Smolarz [11] obtained an analogous result for quasiconvex functions. Namely, he proved that two functions $f, g: I \rightarrow \mathbb{R}$ can be separated by a quasiconvex function if and only if

$$
f(\lambda x+(1-\lambda) y) \leqslant \max \{g(x), g(y)\},
$$

for all $x, y \in I$ and $\lambda \in[0,1]$ (see also [3]).

In this paper we introduce the notion of quasiconvex stochastic processes and present some properties of them. In particular we show that a stochastic process is convex if and only if it is Jensen-convex and quasiconvex. Our main result extends the Smolarz separation theorem to quasiconvex stochastic 
processes. As a consequence we obtain a Hyers-Ulam-type stability result for quasiconvex stochastic processes.

\section{Preliminaries}

Let $(\Omega, \mathcal{A}, P)$ be an arbitrary probability space and $I \subset \mathbb{R}$ be an interval. A function $X: \Omega \rightarrow \mathbb{R}$ is called a random variable, if it is $\mathcal{A}$-measurable. A function $X: I \times \Omega \rightarrow \mathbb{R}$ is called a stochastic process, if for every $t \in I$ the function $X(t, \cdot)$ is a random variable.

Recall that a stochastic process $X: I \times \Omega \rightarrow \mathbb{R}$ is said to be convex, if

$$
\left.X\left(\lambda t_{1}+(1-\lambda) t_{2}, \cdot\right) \leqslant \lambda X\left(t_{1}, \cdot\right)+(1-\lambda) X\left(t_{2}, \cdot\right) \quad \text { (a.e. }\right),
$$

for all $t_{1}, t_{2} \in I$ and $\lambda \in[0,1]$.

A stochastic process $X: I \times \Omega \rightarrow \mathbb{R}$ is called Jensen-convex, if

$$
X\left(\frac{t_{1}+t_{2}}{2}, \cdot\right) \leqslant \frac{X\left(t_{1}, \cdot\right)+X\left(t_{2}, \cdot\right)}{2} \quad \text { (a.e.) }
$$

for all $t_{1}, t_{2} \in I$.

Convex and Jensen-convex stochastic processes were investigated by many authors and various properties and applications of them can be found in the literature (see, for instance, $[5,6,9,10]$ and the references therein).

We say that a stochastic process $X: I \times \Omega \rightarrow \mathbb{R}$ is quasiconvex, if

$$
X\left(\lambda t_{1}+(1-\lambda) t_{2}, \cdot\right) \leqslant \max \left\{X\left(t_{1}, \cdot\right), X\left(t_{2}, \cdot\right)\right\} \quad \text { (a.e.) },
$$

for all $t_{1}, t_{2} \in I$ and $\lambda \in[0,1]$.

We start our investigation with two simple observations.

Observation 1. The following conditions are equivalent:

(i) $X: I \times \Omega \rightarrow \mathbb{R}$ is a quasiconvex stochastic process.

(ii) For every random variable $A: \Omega \rightarrow \mathbb{R}$ the level set

$$
L_{A}=\{t \in I: X(t, \cdot) \leqslant A(\cdot) \quad(\text { a.e. })\}
$$

is convex.

Proof. Suppose first that $X$ is a quasiconvex stochastic process and fix a random variable $A: \Omega \rightarrow \mathbb{R}$. Let $t_{1}, t_{2} \in L_{A}$ and $\lambda \in[0,1]$. By (1) and the definition of level sets we have

$$
\begin{aligned}
X\left(\lambda t_{1}+(1-\lambda) t_{2}, \cdot\right) & \leqslant \max \left\{X\left(t_{1}, \cdot\right), X\left(t_{2}, \cdot\right)\right\} \\
& \leqslant \max \{A(\cdot), A(\cdot)\}=A(\cdot) \quad \text { (a.e. }) .
\end{aligned}
$$

Thus $\lambda t_{1}+(1-\lambda) t_{2} \in L_{A}$, which proves that $L_{A}$ is convex.

Assume now that the sets $L_{A}$ are convex for all random variables $A$. Fix $t_{1}, t_{2} \in I$ and $\lambda \in[0,1]$. Define $A(\cdot)=\max \left\{X\left(t_{1}, \cdot\right), X\left(t_{2}, \cdot\right)\right\}$. Then, of course, 
$t_{1}, t_{2} \in L_{A}$, and, by the convexity of $L_{A}$, we have $\lambda t_{1}+(1-\lambda) t_{2} \in L_{A}$. It means that the inequality

$$
X\left(\lambda t_{1}+(1-\lambda) t_{2}, \cdot\right) \leqslant A(\cdot)=\max \left\{X\left(t_{1}, \cdot\right), X\left(t_{2}, \cdot\right)\right\} \quad \text { (a.e.) }
$$

holds and $X$ is quasiconvex.

Observation 2. If a stochastic process $X: I \times \Omega \rightarrow \mathbb{R}$ is convex, then it is quasiconvex.

Proof. By the convexity of $X$, for all $t_{1}, t_{2} \in I$ and $\lambda \in[0,1]$, we have

$$
\begin{aligned}
X\left(\lambda t_{1}+(1-\lambda) t_{2}, \cdot\right) & \leqslant \lambda X\left(t_{1}, \cdot\right)+(1-\lambda) X\left(t_{2}, \cdot\right) \\
& \leqslant \max \left\{X\left(t_{1}, \cdot\right), X\left(t_{2}, \cdot\right)\right\} \quad \text { (a.e.) },
\end{aligned}
$$

which shows that the process $X$ is quasiconvex.

Clearly, quasiconvex (as well as Jensen-convex) stochastic processes need not be convex. However, if a stochastic proces is both quasiconvex and Jensenconvex, then it is convex.

Proposition 3. Let $I$ be an open interval. A stochastic process $X: I \times \Omega \rightarrow \mathbb{R}$ is convex if and only if it is quasiconvex and Jensen-convex.

Proof. The "only if" part is clear. To prove the "if" part fix $t_{1}, t_{2} \in I, t_{1}<t_{2}$. By the quasiconvexity of $X$, for every $t \in\left[t_{1}, t_{2}\right]$, we have

$$
X(t, \cdot) \leqslant \max \left\{X\left(t_{1}, \cdot\right), X\left(t_{2}, \cdot\right)\right\} \quad \text { (a.e.). }
$$

This implies that the process $X$ is $P$-upper bounded on $\left[t_{1}, t_{2}\right]$, that is

$$
\lim _{n \rightarrow \infty} \sup _{t \in\left[t_{1}, t_{2}\right]}\{P(\{\omega \in \Omega:|X(t, \omega)| \geq n\})\}=0 .
$$

Since $X$ is also Jensen-convex, it follows, by the Bernstein-Doetsch-type theorem, that $X$ is continuous in probability and, consequently, convex (see [6] Theorems 4, 5).

\section{Main result}

At the beginning of this section we would like to recall the definition and basic properties of the essential infimum of a collection of functions. Let $(\Omega, \mathcal{F}, \mu)$ be a measure space and $\mathcal{S}$ be a collection of measurable functions $f: \Omega \rightarrow \mathbb{R}$. On $\mathbb{R}$ the Borel $\sigma$-algebra is used. If $\mathcal{S}$ is a countable set, then we may define the pointwise infimum of the functions from $\mathcal{S}$, which will itself be measurable. If $\mathcal{S}$ is uncountable, then the pointwise infimum need not be measurable. In this case, the essential infimum can be used. The essential infimum of $\mathcal{S}$, written as ess $\inf \mathcal{S}$, if it exists, is a measurable function $f: \Omega \rightarrow \mathbb{R}$ satisfying the two following axioms:

- $f \leqslant g$ almost everywhere, for any $g \in \mathcal{S}$, 
- if $h: \Omega \rightarrow \mathbb{R}$ is measurable and $h \leqslant g$ almost everywhere for every $g \in \mathcal{S}$, then $h \leqslant f$ almost everywhere.

Note that if $f$ is the essential infimum and $g: \Omega \rightarrow \mathbb{R}$ is equal to $f$ almost everywhere, then $g$ is also an essential infimum. Conversely, if $f$ and $g$ are both essential infima, then, from the above definition $f \leqslant g$ and $g \leqslant f$, so $f=g$ almost everywhere. It can be shown that for a $\sigma$-finite measure $\mu$, the essential infimum of $\mathcal{S}$ does exist. Furthermore, there exists a sequence $\left(f_{n}\right)_{n \in \mathbb{N}}$ in $\mathcal{S}$ such that

$$
\text { ess } \inf \mathcal{S}=\inf \left\{f_{n}: n \in \mathbb{N}\right\} .
$$

For more details we refer the reader to [2].

The following properties of essential infimum will be useful in the sequel.

Lemma 4. Let $(\Omega, \mathcal{F}, \mu)$ be a $\sigma$-finite measure space, $\mathcal{S}$ be a nonempty collection of measurable real functions defined on $\Omega$, and let $g: \Omega \rightarrow \mathbb{R}$ be a measurable function. If ess inf $\mathcal{S}<g$ almost everywhere, then there exist sets $\Omega_{n} \in \mathcal{F}$ and functions $f_{n} \in \mathcal{S}$ for $n \in \mathbb{N}$, such that $\mu\left(\Omega \backslash \bigcup_{n \in \mathbb{N}} \Omega_{n}\right)=0$ and $f_{n}<g$ on $\Omega_{n}, n \in \mathbb{N}$.

Proof. By the fact mentioned above there exists a sequence $\left(f_{n}\right)_{n \in \mathbb{N}}$ of elements of $\mathcal{S}$ such that

$$
\operatorname{ess} \inf \mathcal{S}=\inf \left\{f_{n}: n \in \mathbb{N}\right\}
$$

Assume that $\inf \left\{f_{n}: n \in \mathbb{N}\right\}<g$ on $\bar{\Omega}=\Omega \backslash \Omega_{0}$, where $\mu\left(\Omega_{0}\right)=0$. By the definition of the infimum, for every $\omega \in \bar{\Omega}$ there exists $n \in \mathbb{N}$ such that $f_{n}(\omega)<g(\omega)$.

Define the sets

$$
\Omega_{n}=\left\{\omega \in \bar{\Omega}: f_{n}(\omega)<g(\omega)\right\} .
$$

Then $\Omega_{n} \in \mathcal{F}, \bigcup_{n \in \mathbb{N}} \Omega_{n}=\bar{\Omega}$, and $f_{n}<g$ on $\Omega_{n}$ for $n \in \mathbb{N}$, which was to be proved.

Lemma 5. Let $f: \Omega \rightarrow \mathbb{R}$ be a measurable function and $\mathcal{S}$ be a family of all measurable functions $g: \Omega \rightarrow \mathbb{R}$ such that $f<g$ almost everywhere. Then ess $\inf \mathcal{S}=f$.

Proof. Clearly ess inf $\mathcal{S} \geqslant f$ almost everywhere. To conclude the proof it is enough to observe that $f+\frac{1}{n} \in \mathcal{S}$ for all $n \in \mathbb{N}$.

Now we present our main theorem. It gives a condition under which two stochastic processes can be separated by a quasiconvex stochastic process.

Theorem 6. Let $X, Y: I \times \Omega \rightarrow \mathbb{R}$ be stochastic processes. There exists a quasiconvex stochastic process $H: I \times \Omega \rightarrow \mathbb{R}$ such that

$$
X(t, \cdot) \leqslant H(t, \cdot) \leqslant Y(t, \cdot) \quad \text { (a.e.), } \quad t \in I,
$$


if and only if

$$
X\left(\lambda t_{1}+(1-\lambda) t_{2}, \cdot\right) \leqslant \max \left\{Y\left(t_{1}, \cdot\right), Y\left(t_{2}, \cdot\right)\right\} \quad \text { (a.e.) }
$$

for all $t_{1}, t_{2} \in I$ and $\lambda \in[0,1]$.

Proof. The sufficiency is obvious. To prove the necessity assume that $X$ and $Y$ fulfil (2). Given a random variable $A: \Omega \rightarrow \mathbb{R}$ consider the level set

$$
L_{A}=\{t \in I: Y(t, \cdot) \leqslant A(\cdot) \quad \text { (a.e.) }\} .
$$

Let $C_{A}=\operatorname{conv} L_{A}$ denote the convex hull of the set $L_{A}$. Define a stochastic process $H: I \times \Omega \rightarrow \mathbb{R}$ by

$$
H(t, \cdot)=\operatorname{ess} \inf \left\{A: t \in C_{A}\right\} .
$$

Fix $t_{0} \in I$ and take a random variable $A: \Omega \rightarrow \mathbb{R}$ such that $t_{0} \in C_{A}$. In view of the Caratheodory theorem (cf. [8]) we have $t_{0}=\lambda t_{1}+(1-\lambda) t_{2}$, for some $t_{1}, t_{2} \in L_{A}$ and $\lambda \in[0,1]$. Hence $Y\left(t_{1}, \cdot\right) \leqslant A(\cdot) \quad$ (a.e.) and $Y\left(t_{2}, \cdot\right) \leqslant$ $A(\cdot) \quad$ (a.e.) and, by inequality (2), we obtain

$$
X\left(t_{0}, \cdot\right)=X\left(\lambda t_{1}+(1-\lambda) t_{2}, \cdot\right) \leqslant \max \left\{Y\left(t_{1}, \cdot\right), Y\left(t_{2}, \cdot\right)\right\} \leqslant A(\cdot) \quad \text { (a.e.). }
$$

Since the above inequality holds for any random variable $A$, such that $t_{0} \in C_{A}$, we get

$$
X\left(t_{0}, \cdot\right) \leqslant \operatorname{ess} \inf \left\{A: t_{0} \in C_{A}\right\}=H\left(t_{0}, \cdot\right) \quad \text { (a.e.). }
$$

Moreover, since for every fixed $t_{0} \in I$ we have $t_{0} \in L_{Y\left(t_{0}, \cdot\right)} \subset C_{Y\left(t_{0}, \cdot\right)}$, we get also

$$
H\left(t_{0}, \cdot\right)=\operatorname{ess} \inf \left\{A: \quad t_{0} \in C_{A}\right\} \leqslant Y\left(t_{0}, \cdot\right) \quad \text { (a.e.). }
$$

Now we will show that the stochastic process $H$ is quasiconvex. Fix $t_{1}, t_{2} \in I$ and $\lambda \in[0,1]$. The following cases are possible:

(i) $H\left(t_{1}, \cdot\right) \leqslant H\left(t_{2}, \cdot\right) \quad$ (a.e.).

(ii) $H\left(t_{1}, \cdot\right)>H\left(t_{2}, \cdot\right) \quad$ (a.e.).

(iii) The following sets $\Omega_{1}$ and $\Omega_{2}$ have positive measure

$$
\begin{aligned}
& \Omega_{1}=\left\{\omega: H\left(t_{1}, \omega\right) \leqslant H\left(t_{2}, \omega\right)\right\}, \\
& \Omega_{2}=\left\{\omega: H\left(t_{1}, \omega\right)>H\left(t_{2}, \omega\right)\right\} .
\end{aligned}
$$

Assume first that case (i) holds. We will show that there exist sets $\Omega_{n, k} \in$ $\mathcal{A}, n, k \in \mathbb{N}$, such that $P\left(\bigcup_{n, k \in \mathbb{N}} \Omega_{n, k}\right)=1$ and for all $n, k \in \mathbb{N}, H\left(\lambda t_{1}+(1-\right.$ ג) $\left.t_{2}, \cdot\right) \leqslant H\left(t_{2}, \cdot\right)$ almost everywhere on $\Omega_{n, k}$.

Take an arbitrary random variable $B: \Omega \rightarrow \mathbb{R}$ satisfying

$$
H\left(t_{2}, \cdot\right)=\operatorname{ess} \inf \left\{A: t_{2} \in C_{A}\right\}<B(\cdot) \quad \text { (a.e.). }
$$


By Lemma 4 there exist sets $\Omega_{n} \in \mathcal{A}$ and random variables $A_{n} \in\left\{A: t_{2} \in\right.$ $\left.C_{A}\right\}$ such that $P\left(\bigcup_{n \in \mathbb{N}} \Omega_{n}\right)=1$ and for every $n \in \mathbb{N}, A_{n}<B$ on $\Omega_{n}$. Then

$$
\begin{aligned}
& t_{2} \in C_{A_{n}}=\operatorname{conv}\left\{t \in I: Y(t, \cdot) \leqslant A_{n}(\cdot) \quad \text { (a.e.) }\right\} \\
& \subset \operatorname{conv}\left\{t \in I: Y(t, \cdot)<B(\cdot) \quad \text { (a.e.) on } \Omega_{n}\right\} .
\end{aligned}
$$

Now, we use the fact that $H\left(t_{1}, \cdot\right) \leqslant H\left(t_{2}, \cdot\right)<B(\cdot) \quad$ (a.e.) and apply Lemma 4 separately for every set $\Omega_{n}, n \in \mathbb{N}$. There exist sets $\Omega_{n, k} \subset \Omega_{n}, \Omega_{n, k} \in \mathcal{A}$ and random variables $A_{n, k} \in\left\{A: t_{1} \in C_{\left.A\right|_{\Omega_{n}}}\right\}$ such that $P\left(\bigcup_{k \in \mathbb{N}} \Omega_{n, k}\right)=P\left(\Omega_{n}\right)$ and $A_{n, k}<B$ on $\Omega_{n, k}$ for $n, k \in \mathbb{N}$. Then

$$
\begin{aligned}
t_{1} \in C_{\left.A_{n, k}\right|_{\Omega_{n}}} & =\operatorname{conv}\left\{t \in I: Y(t, \cdot) \leqslant A_{n, k}(\cdot) \quad \text { (a.e.) on } \Omega_{n}\right\} \\
& \subset \operatorname{conv}\left\{t \in I: Y(t, \cdot)<B(\cdot) \quad \text { (a.e.) on } \Omega_{n, k}\right\} .
\end{aligned}
$$

By (4) and (5), for every $n, k \in \mathbb{N}$ we obtain

$$
t_{1}, t_{2} \in \operatorname{conv}\left\{t \in I: Y(t, \cdot)<B(\cdot) \quad \text { (a.e.) on } \Omega_{n, k}\right\}
$$

and consequently

$$
\lambda t_{1}+(1-\lambda) t_{2} \in \operatorname{conv}\left\{t \in I: Y(t, \cdot)<B(\cdot) \quad \text { (a.e.) on } \Omega_{n, k}\right\} .
$$

Hence, by the definition of $H$, we get $H\left(\lambda t_{1}+(1-\lambda) t_{2}, \cdot\right) \leqslant B(\cdot) \quad$ (a.e.) on $\Omega_{n, k}$. Since the family $\left(\Omega_{n, k}\right)_{n, k \in \mathbb{N}}$ is countable and $P\left(\bigcup_{k \in \mathbb{N}} \Omega_{n, k}\right)=P\left(\Omega_{n}\right)$ for $n \in \mathbb{N}$ and $P\left(\bigcup_{n \in \mathbb{N}} \Omega_{n}\right)=1$, we also have $H\left(\lambda t_{1}+(1-\lambda) t_{2}, \cdot\right) \leqslant$ $B(\cdot) \quad$ (a.e.) on $\Omega$. Using the fact, that this inequality holds for every random variable $B$ satisfying (3), by Lemma 5 we obtain

$$
\left.H\left(\lambda t_{1}+(1-\lambda) t_{2}, \cdot\right) \leqslant H\left(t_{2}, \cdot\right)=\max \left\{H\left(t_{1}, \cdot\right), H\left(t_{2}, \cdot\right)\right\} \quad \text { (a.e. }\right) .
$$

This finishes the the proof in case (i).

The proof in case (ii) is analogous, so we omit it.

Now assume that case (iii) holds. We consider two processes $H_{1}$ and $H_{2}$ being the restrictions of $H$ to $I \times \Omega_{1}$ and $I \times \Omega_{2}$, respectively. Then for $H_{1}$ case (i) occurs and for $H_{2}$ case (ii) occurs. It means that

$$
H_{1}\left(\lambda t_{1}+(1-\lambda) t_{2}, \cdot\right) \leqslant \max \left\{H_{1}\left(t_{1}, \cdot\right), H_{1}\left(t_{2}, \cdot\right)\right\} \quad \text { (a.e.) on } \quad \Omega_{1}
$$

and

$$
H_{2}\left(\lambda t_{1}+(1-\lambda) t_{2}, \cdot\right) \leqslant \max \left\{H_{2}\left(t_{1}, \cdot\right), H_{2}\left(t_{2}, \cdot\right)\right\} \quad \text { (a.e.) on } \Omega_{2} .
$$

Consequently

$$
H\left(\lambda t_{1}+(1-\lambda) t_{2}, \cdot\right) \leqslant \max \left\{H\left(t_{1}, \cdot\right), H\left(t_{2}, \cdot\right)\right\} \quad \text { (a.e.) on } \quad \Omega
$$

and the proof is complete. 


\section{Hyers-Ulam stability}

As a direct consequence of Theorem 6 we obtain the following Hyers-Ulamtype stability result for quasiconvex stochastic processes. For the classical Hyers-Ulam theorem see [4]. The stability theorem for quasiconvex functions was obtained in [7] (cf. also [11]).

Theorem 7. Let $\varepsilon$ be a positive constant. If a stochastic process $X: I \times \Omega \rightarrow \mathbb{R}$ satisfies the inequality

$$
X\left(\lambda t_{1}+(1-\lambda) t_{2}, \cdot\right) \leqslant \max \left\{X\left(t_{1}, \cdot\right), X\left(t_{2}, \cdot\right)\right\}+\varepsilon \quad \text { (a.e.) }
$$

for all $t_{1}, t_{2} \in I$ and $\lambda \in[0,1]$, then there exists a quasiconvex stochastic

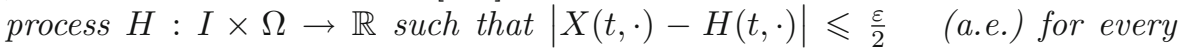
$t \in I$.

Proof. To prove the above theorem it is enough to apply Theorem 6 to the stochastic processes $X$ and $X+\varepsilon$. Hence, there exists a process $H_{1}: I \times \Omega \rightarrow$ $\mathbb{R}$, which is quasiconvex and satisfies $X(t, \cdot) \leqslant H_{1}(t, \cdot) \leqslant X(t, \cdot)+\varepsilon \quad$ (a.e.). By taking $H(t, \cdot)=H_{1}(t, \cdot)-\frac{\varepsilon}{2}$ we get $|X(t, \cdot)-H(t, \cdot)| \leqslant \frac{\varepsilon}{2} \quad$ (a.e.). This completes the proof.

Open Access. This article is distributed under the terms of the Creative Commons Attribution License which permits any use, distribution, and reproduction in any medium, provided the original author(s) and the source are credited.

\section{References}

[1] Baron, K., Matkowski, J., Nikodem, K.: A sandwich with convexity. Math. Pannonica 5/1, 139-144 (1994)

[2] Doob, J.L.: Measure theory. Graduate Texts in Mathematics. Springer, Berlin (1993)

[3] Förg-Rob, W., Nikodem, K., Páles, Zs.: Separation by monotonic functions. Math. Pannonica 7/2, 191-196 (1993)

[4] Hyers, D.H., Ulam, S.M.: Approximately convex functions. Proc. Am. Math. Soc. 3, 821828 (1952)

[5] Kotrys, D.: Hermite-Hadamard inequality for convex stochastic processes. Aequat. Math. 83, 143-151 (2012)

[6] Nikodem, K.: On convex stochastic processes. Aequat. Math. 20, 184-197 (1980)

[7] Nikodem, K.: Approximately quasiconvex functions. C. R. Math. Rep. Acad. Sci. Canada 10, 291-294 (1988)

[8] Roberts, A.W., Varberg, D.E.: Convex functions. Academic Press, New York (1973)

[9] Shaked, M., Shanthikumar, J.G.: Stochastic convexity and its applications. Adv. Appl.Prob. 20, 427-446 (1988)

[10] Skowroński, A.: On some properties of J-convex stochastic processes. Aequat. Math. 44, 249-258 (1992)

[11] Smolarz, J.: On some functional inequalities connected with quasiconvex functions. C. R. Math. Rep. Acad. Sci. Canada 16/6, 241-246 (1994) 
Kazimierz Nikodem

Department of Mathematics and Computer Science

University of Bielsko-Biała

Willowa 2, 43-309 Bielsko-Biała

Poland

e-mail: knikodem@ath.bielsko.pl

Dawid Kotrys

e-mail: dkotrys@gmail.com

Received: February 6, 2014 